\title{
IoT Livestock Estrus Monitoring System based on Machine Learning
}

\author{
Meonghun Lee1)
}

\begin{abstract}
Machine learning is an artificial intelligence technology that analyzes data and builds data-based models, and its role in Internet-of-Things (IoT) applications is growing. In current ranches, artificial insemination is performed to make cows pregnant. Accurate estrus detection of cows is essential for the success in artificial insemination. The traditional method to detect estrus of cows is visual observation, whose success rate largely depends on the observers' experience and the frequency of observation. The integration of IoT and machine learning enables livestock diseases and estrus to be predicted. Livestock activity was measured by acceleration sensors attached to animals. Then, a system was created that can identify atypical symptoms such as those of disease and estrus. To predict calving dates in breeding cows, behavioral patterns were analyzed using machine-learning algorithms. Finally, only simple judgments by owners are required to decide whether artificial insemination will be performed.

Keywords : Internet of Things, Estrus Detection, Machine Learning, Livestock Monitoring, Smart Farm
\end{abstract}

\section{Introduction}

Recently, Web, social networking, and cloud services using wired and wireless networks have led to an exponential increase in various forms of data, and this increase is expected to accelerate further as the Internet of Things (IoT) is adopted. The IoT consists of an infrastructure and services that enable the exchange of information by connecting the physical objects in an environment with the Internet[1][2].

The objects are simply a means of connection, and the real value is in the data collected from these objects, which can be analyzed by businesses and managers for commercial innovation. To effectively integrate various forms of data scattered about the physical and virtual worlds, there is a need to determine what data to collect and what statistical model to create through exploratory data analysis[3][4]. Therefore, machine learning is becoming an increasingly important artificial intelligence technology because it can consider the variation, scale, distribution, and frequency of data generated by the IoT[5][6].

Received(August 4, 2018), Review Result(1st: August 16, 2018, 2nd: September 7, 2018), Accepted(September 10, 2018)

1) (Researcher, Corresponding Author) Dept. of Agricultural Eng., National Institute of Agricultural Sciences, Jeollabuk-do 55365, Republic of Korea

email: leemh5544@gmail.com 
The integration of IoT and livestock management, particularly the use of the Internet and networking technology in existing automation devices, to observe and quantify environmental and animal conditions without limits in time or space, is called livestock smart farming[7][8]. In particular, the observation of estrus and timing of fertilization account for the greatest proportion of livestock breeding management[9][10]. Recently, livestock farms have been experiencing difficulties due to the poor prediction of livestock disease and estrus, and the damage is increasing. Among livestock management technologies, disease and estrus prediction is directly connected to the income of livestock farms, and unsuccessful prediction leads to economic loss.

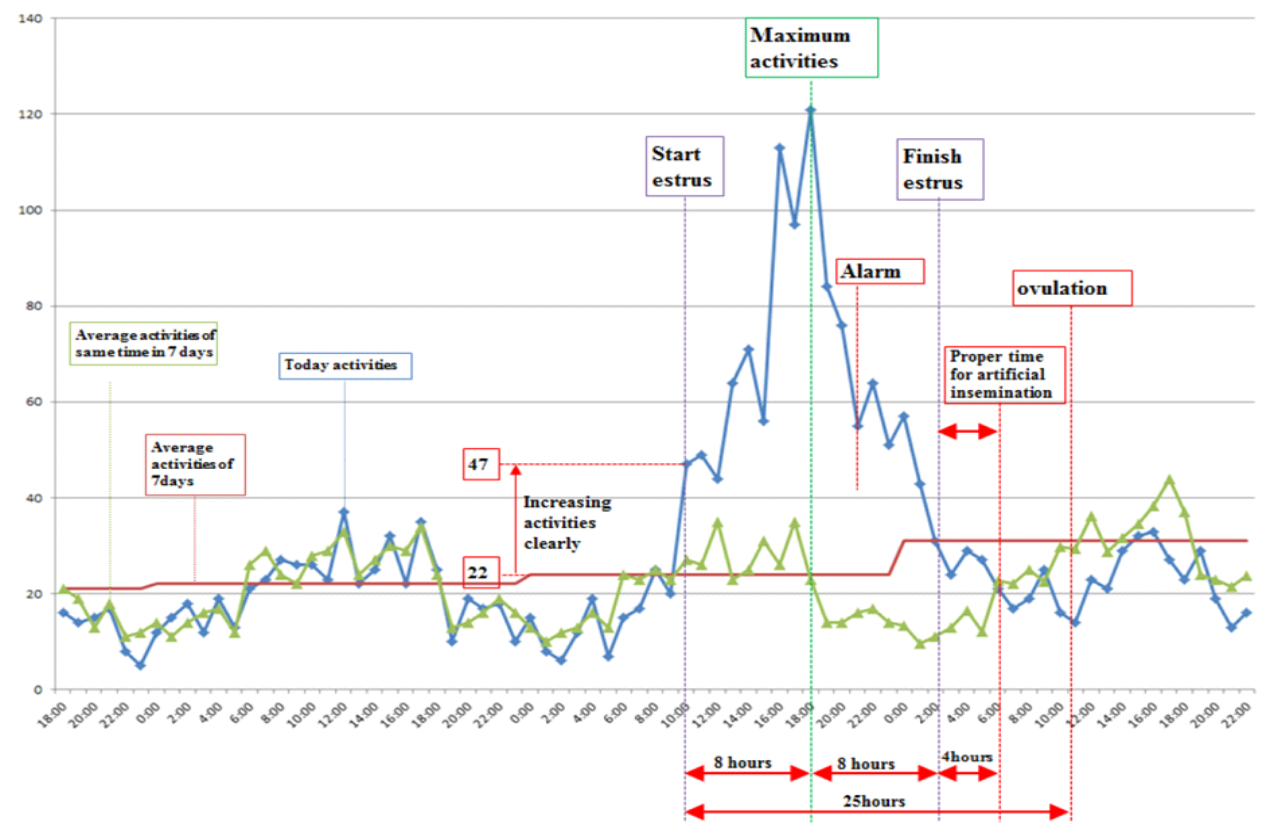

[Fig. 1] Diagram of The Changes in Movement of Breeding Cows Using IoT Sensors

In this study, acceleration sensors were attached to livestock in a farms with poor prediction to measure livestock activity and to analyze the collected to data to enable rapid response in case of atypical symptoms, such as various diseases and estrus, in order to suggest a better system[11][12]. Upon estrus, livestock movement increases above normal, while movement decreases in diseased livestock[13]. Based on these characteristics, a livestock movement monitoring system was designed using an acceleration sensor.

The remainder of this paper is organized as follows. In Section 2, the estrous characteristics of breeding cows and type of analysis used on the acceleration sensor data is discussed, and 
in Section 3, a system for machine-learning analysis of the collected data is described. In Section 4, various machine-learning algorithms are used to analyze certain behavioral patterns from real data to predict calving breeding cows. The paper is concluded in Section 5.

\section{Measuring Estrus Using Acceleration Sensors}

\subsection{Characteristics of Estrus in Breeding Cows}

To increase the reproductive rate of each individual cow, it is important to identify the breeding information of each cow, which consists of final delivery date, number of days since calving, fertilization date, number of fertilizations, number of calvings, and first estrus date since calving, to identify any problems with the livestock farm and manage breeding appropriately.

The system suggested in this paper employs hardware to monitor movement, predict estrus, and hence increase fertilization rate in conjunction with a comprehensive individual management system[14]. The behavioral patterns of cows differ according to the farm environment and breeding management differs according to the management system used at each farm. Therefore, it is necessary to use a specialized breeding management method at each farm. Further, there is a need for the system to detect estrus at night and at times when the farm is not manned.

The observation of estrus symptoms is the focus of cow breeding management. Accurate estrus detection enables the optimum period between breeding to be maintained so that breeding cows can be successfully managed. Upon estrus, cows show the following symptoms. First, estrous cows smell and lick other cows before estrus (6-10 hours), and then accept mounting by other cows. At this point, it is much easier to observe a mounting cow, but the cow being mounted is $65 \%-98 \%$ more likely to be in estrus than the mounting cow. Estrus lasts for about 18 hours, during which the cow allows mounting by other cows, increases her movement, cries frequently, is anxious, slowly avoids approaching people, behaves timidly, and does not consume food. [Fig. 1] shows the changes in movement in conventional breeding cows with respect to estrus determined using biological information. It shows movement over a day, average movement over a week, and average hourly movement over a week for each individual cow. 


\subsection{Characteristics of Estrus in Breeding Cows}

Movement to determine estrus onset was detected using 3-axis acceleration sensors. Equation (1) was used extract features from the sensor signal.

$$
V M \quad x^{2}+y_{i}^{2}+z_{i}^{2},(i=1,2,3, \ldots N)
$$

A 3-axis acceleration sensor uses gravitational acceleration to measure the strength of force in the $x, y$, and $z$-axis directions[15-17].

Therefore, the correspondence of the values of each axis of acceleration changes with breeding cow behavior, and these changes can be used to recognize the cow's movement. However, the rotation component of the data can interfere with accurate behavior recognition, and thus, the following equation is used to minimize the rotation component and improve movement recognition accuracy. The signal vector magnitude (SVM) value cancels out the directional component of the 3-axis acceleration signal with respect to gravity to obtain the magnitude of the signal vector[18].

In this study, the system to measure cow movement using IoT acceleration sensors was developed. The data can be used with other necessary breeding information to make a database for a comprehensive individual cow management system that predicts estrus. The implemented system was tested on a real farm to verify its performance.

\section{Livestock Estrus Monitoring System}

\subsection{IoT Node Design Based on 3-axis Acceleration Sensors}
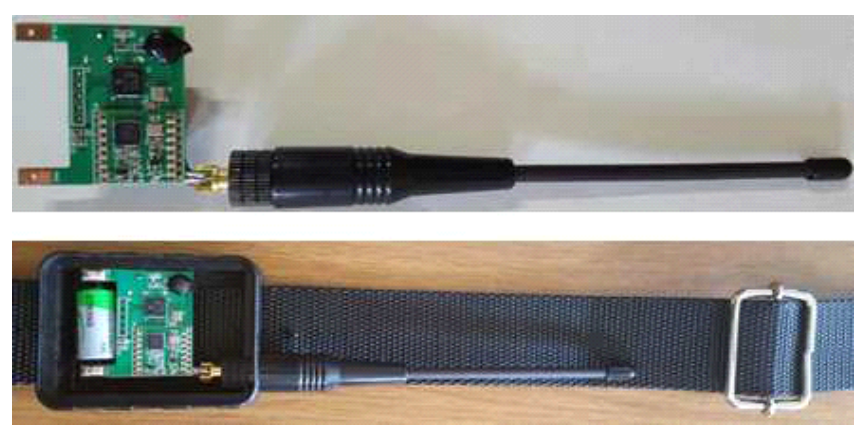

[Fig. 2] The IoT Node Necklce Developed for The Breeding Cows. 
[Fig. 2] shows the IoT node necklace developed for the breeding cows as well as the system for the detection and processing of signals from the low-power acceleration sensors. In the system, the MSP430 microcontroller unit (Texas Instruments, TX, USA) and the MMA7260 3-axis acceleration sensor (Freescale, TX, USA) were used. For system power, a $3.7 \mathrm{~V}$ lithium polymer battery $(250 \mathrm{mAh})$ was used to allow about 43 hours of sustained operation.
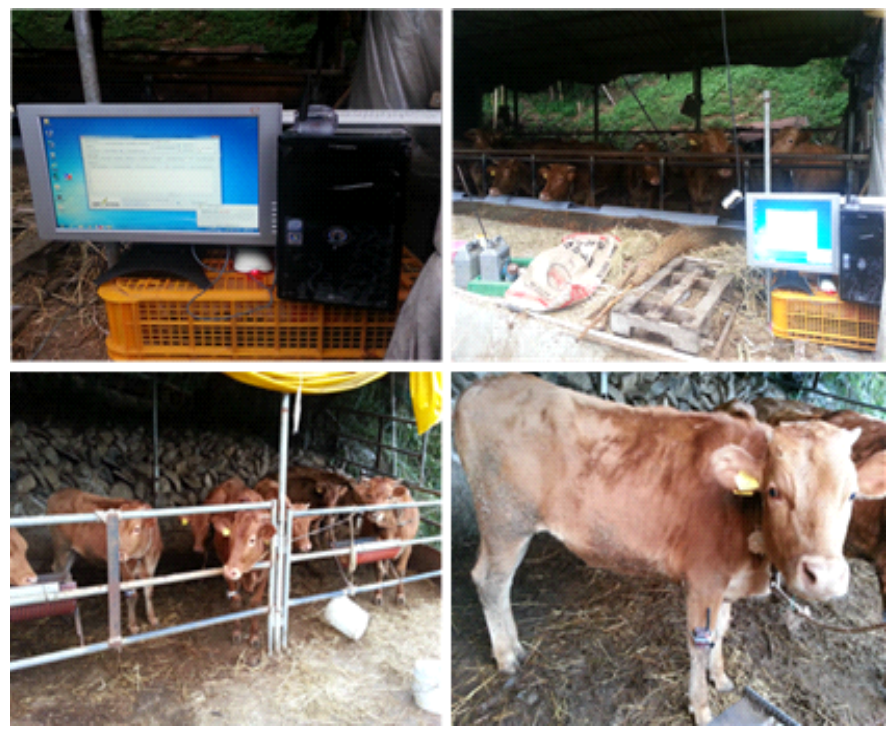

[Fig. 3] The Movement Data Collection System from Breeding Cows that Are Expected to Become Estrous.

[Fig. 3] shows the experimental prototype for the movement data collection from breeding cows that are expected to become estrous. The prototype consists of a movement data storage server, one synchronization node, and two sensor nodes. The two sensor nodes attached to a Korean breeding cow are connected to the synchronization node and the movement information is transmitted using wireless communication in real time to the database sensor.

\subsection{Machine Learning Framework}

[Fig. 4] shows the framework used in this paper, in which an object's data area collected throughout its life using IoT and a machine learning algorithm is used to provide a decision-making service, data product, and more advanced object[19][20].

To manage the data collected from acceleration sensors, formatting and unit conversion was performed manually and they were then stored in the database. The database stores sensor data and livestock activity for analysis in tables. AZURE (Microsoft) was then used to analyze 
the data using an R-based machine learning library[21][22]. A comparison of historical livestock movement information stored in the database and the currently measured livestock movement information is then conducted, which is used to provide a notification service via the Web or smart devices to users in real time. The machine learning prototype developed in this study provides the core technology and tools essential for a data analyst to extract effective business value from a large-scale data set[23][24].

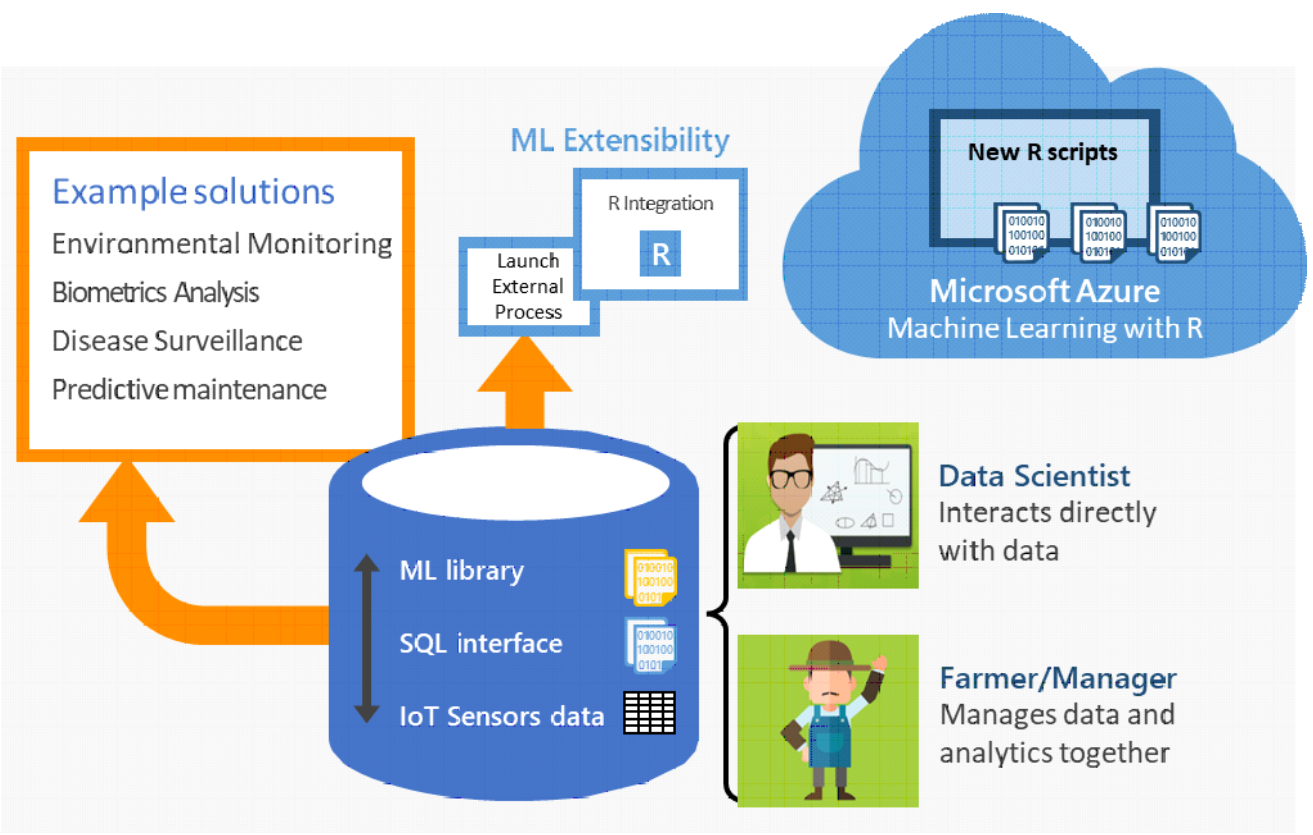

[Fig. 4] Machine Learning Framework for Livestock Estrus Monitoring

\section{Experimental Results}

To predict estrus in breeding cows, data obtained by 3-axis acceleration sensors were analyzed on a cloud server. As shown in [Fig. 1], three patterns (estrus start, peak estrus activities, and estrus finish) were defined from the data features. From these, the behavioral patterns of a breeding cow were analyzed using three machine-learning methods: expectation maximization, random forests, and convolutional neural networks (CNNs)[25-27].

As shown in [Fig. 5], the SVM values substantially differed according to the three behavioral patterns mentioned above. This reflects the increasing gap in SVM value changes collected from the acceleration sensor as the movement of the breeding a cow increases. Although standard deviation changes due to variation in the movement of individual cows and the situation, the 
difference in each behavioral pattern can be seen.

The livestock estrus monitoring system was tested on real breeding cows. To recognize the three behavioral patterns, values were obtained every second from the acceleration sensor and the SVM values were extracted. For the training data for the experiment, 500,000 feature vectors were used; for the test data, 30,000 feature vectors were used.
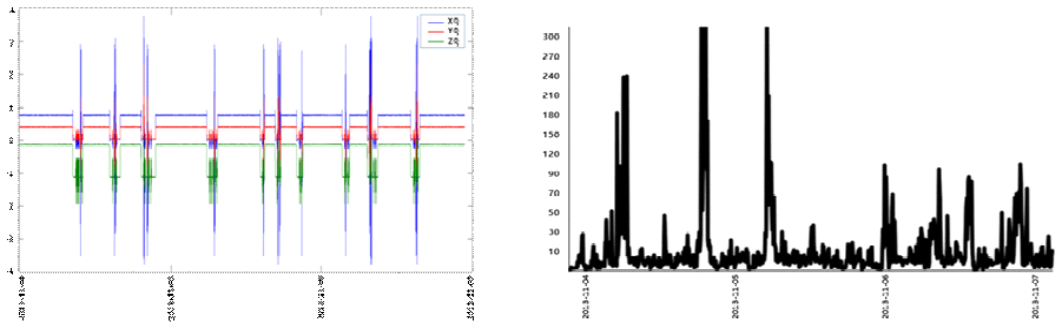

(a) estrus start
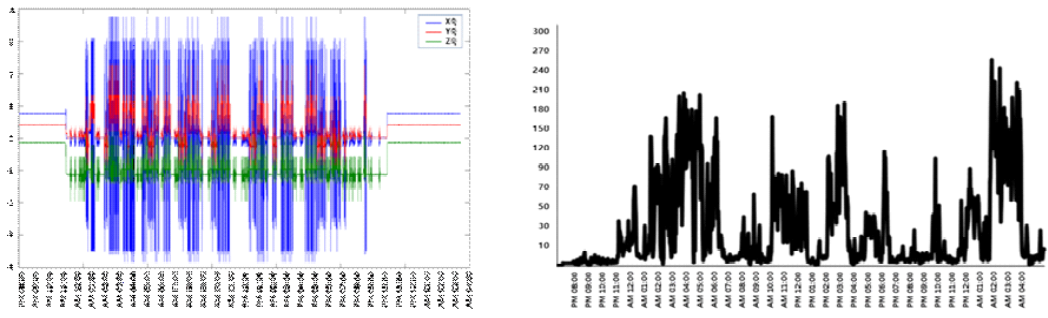

(b) peak estrus activities
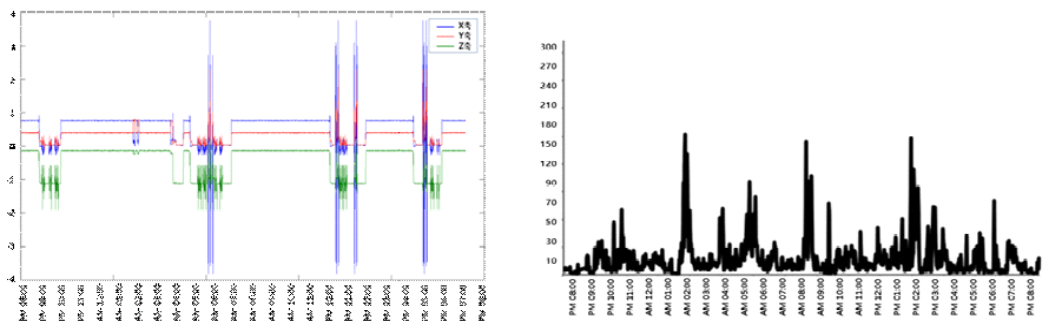

(c) estrus finish

[Fig. 5] Three Patterns of A Breeding Cow (Estrus Start, Peak Estrus Activities, and Estrus Finish)

[Table 1] shows a confusion matrix of the behavioral pattern recognition performances obtained by expectation maximization, random forests, and CNN. Of the three machine-learning algorithms, CNN performed the best. Overall, pattern recognition performance was higher for the estrus finish behavioral pattern because of the more distinct characteristics of its feature vectors. However, estrus finish was often confused with estrus start and peak estrus activities. 
This shows that the behavior of breeding cows and the location of the IoT sensor node substantially influences behavioral pattern recognition in terms of estrus start and peak estrus activities. In the behavior pattern recognition results obtained by $\mathrm{CNN}$, the recognition rates for estrus finish and peak estrus activities were over 98\%, while that for the estrus start behavior was relatively low. The absolute value of the feature vectors indicating estrus start are between the values obtained for peak estrus activities and estrus finish, and thus, the estrus start pattern can be mistaken for these patterns. Further, there is a substantial difference between the feature vectors indicating estrus finish and peak estrus activities. Hence, the two are rarely mistaken for another, as shown in [Table 1].

[Table 1] Confusion Matrix of The Behavioral Pattern Recognition Performances

\begin{tabular}{|c|c|c|c|c|}
\hline \multicolumn{2}{|c|}{ Schedule } & \multirow{2}{*}{$\begin{array}{c}\text { estrus start } \\
73.85\end{array}$} & \multirow{2}{*}{$\begin{array}{c}\text { peak estrus } \\
\text { activities } \\
12.52\end{array}$} & \multirow{2}{*}{$\begin{array}{c}\text { estrus finish } \\
5.63\end{array}$} \\
\hline & estrus start & & & \\
\hline $\begin{array}{l}\text { Expectation } \\
\text { Maximization }\end{array}$ & peak estrus activities & 12.64 & 85.38 & 4.65 \\
\hline & estrus finish & 11.34 & 3.19 & 86.53 \\
\hline \multirow{3}{*}{$\begin{array}{l}\text { Random } \\
\text { Forest }\end{array}$} & estrus start & 82.49 & 5.39 & 12.84 \\
\hline & peak estrus activities & 6.70 & 95.32 & 1.2 \\
\hline & estrus finish & 8.74 & 0 & 91.23 \\
\hline \multirow{3}{*}{$\begin{array}{l}\text { Convolutional } \\
\text { Neural Network }\end{array}$} & estrus start & 73.82 & 6.64 & 17.61 \\
\hline & peak estrus activities & 0.33 & 98.25 & 1.34 \\
\hline & estrus finish & 1.50 & 0 & 98.95 \\
\hline
\end{tabular}

\section{Conclusion}

This study proposed and implemented a system to analyze the 3-axis acceleration information from IoT sensors and compared the pattern-recognition performance of three machine-learning algorithms, expectation maximization, random forests, and $\mathrm{CNN}$, for three behavioral patterns of breeding cows, estrus start, peak estrus activities, and estrus finish. In particular, the CNN achieved outstanding performance compared to the conventional machine-learning methods in the experiments. These results indicate that the signal magnitudes (without rotational features) from the acceleration sensors can be analyzed with respect time to determine estrus start, peak estrus activities, and estrus finish patterns. With these patterns, the calving date of breeding 
cows can be predicted.

The movement of cows in estrus compared to that of non-estrous cows was shown to increase for a certain time period, and the suggested system monitors this movement real time to provide an estrus notification service. Therefore, this system has the potential to accurately identify estrus periods in breeding cows so that the time gap between calving can be decreased, which in turn decreases feed waste. In the future, movement information for diseases as well as estrus will be collected and used to increase the accuracy and reliability of the system. Moreover, the movement information for each animal will be extracted to establish a system applicable to each individual cow.

\section{References}

[1] L. Atzori, A. Iera, G. Morabito, The internet of things: A survey, Computer networks, (2010), Vol.54, pp.2787-2805.

[2] R. Barga, V. Fontama, W. H. Tok, L. Cabrera-Cordon, Predictive analytics with Microsoft Azure machine learning, Springer, (2015)

[3] M. J. Berry, G. Linoff, Data mining techniques: for marketing, sales, and customer support, John Wiley \& Sons, Inc., (1997)

[4] A. Candel, V. Parmar, E. LeDell, A. Arora, H2O, (2015)

[5] H. Cho, S. Kim, J. Baek, and P.S. Fisher, Motion recognition with smart phone embedded 3-axis accelerometer sensor, Proc. IEEE International Conference on System, Man, and Cybernetics (SMC), (2012), October 14-17; Seoul, South Korea, pp.919-924.

[6] M. Copeland, J. Soh, A. Puca, M. Manning, D. Gollob, in Microsoft Azure, Springer, (2015)

[7] C. Cornou, S. Lundbye-Christensen, A. R. Kristensen, Modelling and monitoring sows' activity types in farrowing house using acceleration data, Computers and electronics in agriculture, (2011), Vol.76, pp.316-324.

[8] R. W. DeVaul, S. Dunn, Real-time motion classification for wearable computing applications, 2001 Project Paper, (2001)

[9] U. M. Fayyad, G. Piatetsky-Shapiro, P. Smyth, R. Uthurusamy, Advances in knowledge discovery and data mining, AAAI press Menlo Park, (1996), Vol.21

[10] A. Frost, A review of livestock monitoring and the need for integrated systems, Computers and electronics in agriculture, (1997), Vol.17, pp.139-159.

[11] Y.-J. Hong, I.-J. Kim, S. C. Ahn, H.-G. Kim, Mobile health monitoring system based on activity recognition using accelerometer, Simulation Modelling Practice and Theory, (2010), Vol.18, pp.446-455. 
[12] K. Hornik, C. Buchta, A. Zeileis, Open-source machine learning: R meets Weka, Computational Statistics, (2009), Vol.24, pp.225-232.

[13] K.-Z. Huang, H. Yang, I. King, M. R. Lyu, Machine learning: modeling data locally and globally, Springer Science \& Business Media, (2008)

[14] H. Kashyap, H. A. Ahmed, N. Hoque, S. Roy, D. K. Bhattacharyya, Big data analytics in bioinformatics: A machine learning perspective, (2015), arXiv preprint arXiv:1506.05101

[15] N. Ketkar, in Deep Learning with Python, Springer, (2017), pp.61-76.

[16] H. Kim, H. Yoe, Design and Implementation of ICT-Based System for Information Management of Livestock Farm, International Journal of Smart Home, (2014), Vol.8, pp.1-6.

[17] Y. Kodratoff, R. S. Michalski, Machine learning: an artificial intelligence approach, Morgan Kaufmann, (2014), Vol.3

[18] M. Lee, J. Hwang, and H. Yoe, Agricultural production system based on IoT, IEEE 16th International Conference on Computational Science and Engineering (CSE), (2013), December 3-5; Sydney, NSW, Australia, pp.833-837.

[19] M.-H. Lee, H. Yoe, Design of Agro-Livestock Products Traceability System using EPC Network, Journal of the Korea Institute of Information and Communication Engineering, (2007), Vol.11, pp.216-221.

[20] A. Liaw, M. Wiener, Classification and regression by randomForest, R news, (2002), Vol.2, pp.18-22.

[21] Y. Ling, L. Caixing, H. Tiansheng, Z. Haoen, K. H. Kwong, Design of system for monitoring dairy cattle's behavioral features based on wireless sensor networks, Transactions of the Chinese Society of Agricultural Engineering, (2010)

[22] R. S. Michalski, J. G. Carbonell, T. M. M. Learning, An Artificial Intelligence Approach, Understanding the Nature of Learning, (1983), Vol.2, pp.3-26.

[23] T. K. Moon, The expectation-maximization algorithm, IEEE Signal processing magazine, (1996), Vol.13, pp.47-60.

[24] C. Rahe, R. Owens, J. Fleeger, H. Newton, P. Harms, Pattern of plasma luteinizing hormone in the cyclic cow: dependence upon the period of the cycle, Endocrinology, (1980), Vol.107, pp.498-503.

[25] B. Robert, B. White, D. Renter, R. Larson, Evaluation of three-dimensional accelerometers to monitor and classify behavior patterns in cattle, Computers and Electronics in Agriculture, (2009), Vol.67, pp.80-84.

[26] P. Senger, The estrus detection problem: new concepts, technologies, and possibilities, Journal of dairy science, (1994), Vol.77, pp.2745-2753.

[27] R. H. Weber, R. Weber, Internet of things, springer, (2010), Vol.12 